\title{
Convergence and weaker control conditions for hybrid iterative algorithms
}

\author{
Shuang Wang
}

\section{Correspondence:}

wangshuang19841119@163.com School of Mathematical Sciences, Yancheng Teachers University, Yancheng, 224051 Jiangsu, PR China,

\begin{abstract}
Very recently, Yao et al. (Appl. Math. Comput. 216, 822-829, 2010) have proposed a hybrid iterative algorithm. Under the parameter sequences satisfying some quite restrictive conditions, they derived a strong convergence theorem in a Hilbert space. In this article, under the weaker conditions, we prove the strong convergence of the sequence generated by their iterative algorithm to a common fixed point of an infinite family of nonexpansive mappings, which solves a variational inequality. It is worth pointing out that we use a new method to prove our results. An appropriate example, such that all conditions of this result that are satisfied and that other conditions are not satisfied, is provided. Furthermore, we also give a weak convergence theorem for their iterative algorithm involving an infinite family of nonexpansive mappings in a Hilbert space.
\end{abstract}

MSC: 47H05, 47H09, 47H10

Keywords: Strong convergence, Variational inequality, Fixed points, k-Lipschitzian, $\eta$-strongly monotone, Hilbert space

\section{Introduction}

Let $H$ be a real Hilbert space and $C$ be a nonempty, closed, convex subset of $H$, let $F$ : $H \rightarrow H$ be a nonlinear operator. The variational inequality problem is formulated as finding a point $x^{*} \in C$ such that

$$
\left\langle F x^{*}, v-x^{*}\right\rangle \geq 0, \quad \forall v \in C .
$$

In 1964, Stampacchia [1] introduced and studied variational inequality initially. It is now well known that variational inequalities cover as diverse disciplines as partial differential equations, optimal control, optimization, mathematical programming, mechanics, and finance [1-5].

It is known that a mapping $T: H \rightarrow H$ is said to be nonexpansive if $\|T x-T y\| \leq \| x$ - $y \|, \forall x, y \in H$. We use $F(T)$ to denote the set of fixed points of $T$, that is $F(T)=\{x$ $\in H: T x=x\}$.

Yamada [2] introduced the following hybrid iterative method for solving the variational inequality:

$$
x_{n+1}=T x_{n}-\mu \lambda_{n} F\left(T x_{n}\right), \quad n \geq 0,
$$

\section{SpringerOpen ${ }^{\circ}$}

(c) 2011 Wang; licensee Springer. This is an Open Access article distributed under the terms of the Creative Commons Attribution License (http://creativecommons.org/licenses/by/2.0), which permits unrestricted use, distribution, and reproduction in any medium, provided the original work is properly cited. 
where $F$ is a $k$-Lipschitzian and $\eta$-strongly monotone operator with $k>0, \eta>0$ and $0<\mu<2 \eta / k^{2}$. Let a sequence $\left\{\lambda_{n}\right\}$ of real numbers in $(0,1)$ satisfy the conditions below:
(C1) $\lim _{n \rightarrow \infty} \lambda_{n}=0$,
(C2) $\sum_{n=0}^{\infty} \lambda_{n}=\infty$,
(C3) $\lim _{n \rightarrow \infty}\left(\lambda_{n}-\lambda_{n+1}\right) / \lambda_{n+1}^{2}=0$.

He has proved that $\left\{x_{n}\right\}$ generated by (1.1) converges strongly to the unique solution of the variational inequality:

$$
\langle F \tilde{x}, x-\tilde{x}\rangle \geq 0, \quad \forall x \in F(T) .
$$

An example of sequence $\left\{\lambda_{n}\right\}$ which satisfies conditions (C1)-(C3) is given by $\lambda_{n}=1 /$ $n^{\sigma}$, where $0<\sigma<1$. We note that the condition (C3) was first used by Lions [3]. It was observed that Lion's conditions on the sequence $\left\{\lambda_{n}\right\}$ excluded the canonical choice $\lambda_{n}$ $=1 / \mathrm{n}$. This was overcome in 2003 by $\mathrm{Xu}$ and $\operatorname{Kim}[4]$, if $\left\{\lambda_{n}\right\}$ satisfies conditions (C1), (C2), and (C4)

$$
\text { (C4) : } \lim _{n \rightarrow \infty} \lambda_{n} / \lambda_{n+1}=1, \quad \text { or equivalently, } \quad \lim _{n \rightarrow \infty}\left(\lambda_{n}-\lambda_{n+1}\right) / \lambda_{n+1}=0
$$

who proved the strong convergence of $\left\{x_{n}\right\}$ to the unique solution $u^{*}$ of the variational inequality $\left\langle F u^{*}, v-u^{*}\right\rangle \geq 0, \forall v \in C$. It is easy to see that the condition (C4) is strictly weaker than condition (C3), coupled with conditions (C1) and (C2). Moreover, (C4) includes the important and natural choice $\{1 / n\}$ of $\left\{\lambda_{n}\right\}$.

Very recently, motivated by Xu and Kim [4], Yao et al. [5] considered the following algorithms: for $x_{0} \in H$ arbitrarily,

$$
\left\{\begin{array}{l}
y_{n}=x_{n}-\lambda_{n} F\left(x_{n}\right), \\
x_{n+1}=\left(1-\alpha_{n}\right) y_{n}+\alpha_{n} W_{n} y_{n}, n \geq 0,
\end{array}\right.
$$

where $F$ is a $k$-Lipschitzian and $\eta$-strongly monotone operator on $H$, and $W_{n}$ is a $W$ mapping defined by (2.3) cited later. Take $k \in[1, \infty), \eta \in(0,1)$, and $\left\{\lambda_{n}\right\}$ satisfying the conditions $(\mathrm{C} 1)$ and $(\mathrm{C} 2)$. If a sequence $\left\{\alpha_{n}\right\}$ satisfying $(\mathrm{C} 5)$

$$
\text { (C5) : } \alpha_{n} \in\left[\gamma, \frac{1}{2}\right] \text { for some } \gamma>0 \text {, }
$$

then they proved that the sequences $\left\{x_{n}\right\}$ and $\left\{y_{n}\right\}$ defined by (1.2) converge strongly to $x^{*} \in \cap_{n=1}^{\infty} F\left(T_{n}\right)$, which solves the following variational inequality:

$$
\left\langle F x^{*}, x-x^{*}\right\rangle \geq 0, \quad \forall x \in \cap_{n=1}^{\infty} F\left(T_{n}\right) .
$$

We remind the reader of the fact that in order to guarantee the strong convergence of the iterative sequence $\left\{x_{n}\right\}$, there is at least one parameter sequence converging to zero (i.e., $\lambda_{n} \rightarrow 0$ ) as a result of Yamada [2], Xu and Kim [4, Theorem 3.1, and Theorem 3.2] and Yao et al. [5, Theorem 3.2]. In addition, $\eta \in(0,1)$ and (C5) are quite restrictive assumptions in Yao et al. [5].

In this article, under the convergence of no parameter sequences to zero and the weaker conditions on $\alpha_{\mathrm{n}}$ and $\eta$, we prove that the sequence $\left\{y_{n}\right\}$ generated by the 
iterative algorithm (1.2) converges to a common fixed point of an infinite family of nonexpansive mappings, which solves the variational inequality $\left\langle F x^{*}, u-x^{*}\right\rangle \geq 0$, $\forall u \in \cap_{n=1}^{\infty} F\left(T_{n}\right)$. In the meantime, we illustrate that this result is more general than Theorem 3.2 of Yao et al. [5]. That is, we give an appropriate example such that all conditions of this result are satisfied and the conditions $\eta \in(0,1),(\mathrm{C} 1)$, and (C5) in Yao et al. [5, Theorem 3.2] are not satisfied. Furthermore, we also give a weak convergence theorem for hybrid iterative algorithm (1.2) involving an infinite family of nonexpansive mappings in a Hilbert space $H$. It is worth pointing out that we use a new method to prove our main results. The results presented in this article can be viewed as the improvement, supplement, and extension of the results obtained in [2-5].

\section{Preliminaries}

Let $H$ be a real Hilbert space with inner product $\langle\cdot, \cdot\rangle$ and norm $\|\cdot\|$. For the sequence $\left\{x_{n}\right\}$ in $H$, we write $x_{n}-x$ to indicate that the sequence $\left\{x_{n}\right\}$ converges weakly to $x$. $x_{n} \rightarrow x$ implies that $\left\{x_{n}\right\}$ converges strongly to $x$. We denote by $\omega_{w}\left(x_{n}\right)$ the weak $\omega$-limit set of $\left\{x_{n}\right\}$, that is

$$
\omega_{w}\left(x_{n}\right)=\left\{x \in H: x_{n_{i}} \rightarrow x \text { for some subsequence }\left\{x_{n_{i}}\right\} \text { of }\left\{x_{n}\right\}\right\} .
$$

A mapping $F: H \rightarrow H$ is called $k$-Lipschitzian if there exists a positive constant $k$ such that

$$
\|F x-F y\| \leq k|| x-y \|, \quad \forall x, y \in H .
$$

$F$ is said to be $\eta$-strongly monotone if there exists a positive constant $\eta$ such that

$$
\langle F x-F y, x-y\rangle \geq \eta\|x, y\|^{2}, \quad \forall x, y \in H .
$$

It is known that $X$ satisfies Opial's property [6] provided, for each sequence $\left\{x_{n}\right\}$ in $X$, the condition $x_{n} \rightarrow x$ implies

$$
\limsup _{n \rightarrow \infty}\left\|x_{n}-x\right\|<\limsup _{n \rightarrow \infty}\left\|x_{n}-y\right\|, \quad \forall y \in X, y \neq x .
$$

It is known that each $\lambda^{p}(1 \leq p<\infty)$ enjoys this property, while $L^{p}$ does not unless $p=2$.

Finally, it is known that in a Hilbert space, there holds the following equality

$$
\|\lambda x+(1-\lambda) y\|^{2}=\lambda\|x\|^{2}+(1-\lambda)\|y\|^{2}-\lambda(1-\lambda)\|x-y\|^{2}
$$

for all $x, y \in H$ and $\lambda \in[0,1]$ (see Takahashi [7]).

In order to prove our main results, we need the following lemmas:

Lemma 2.1. [8]. Let $H$ be a Hilbert space, $C$ a closed, convex subset of $H$, and $T$ : $C \rightarrow C$ a nonexpansive mapping with $F(T) \neq \varnothing$; if $\left\{x_{n}\right\}$ is a sequence in $C$ weakly converging to $\times$ and if $\left\{(I-T) x_{n}\right\}$ converges strongly to $y$, then $(I-T) x=y$.

Lemma 2.2. [9]. Let $\left\{x_{n}\right\}$ and $\left\{z_{n}\right\}$ be bounded sequences in Banach space $E$ and $\left\{\gamma_{n}\right\}$ be a sequence in $[0,1]$ which satisfies the following condition:

$$
0<\liminf _{n \rightarrow \infty} \gamma_{n} \leq \limsup _{n \rightarrow \infty} \gamma_{n}<1 .
$$

Suppose that $x_{n+1}=\gamma_{n} x_{n}+\left(1-\gamma_{n}\right) z_{n}, n \geq 0$, and $\lim \sup _{n \rightarrow \infty}\left(|| z_{n+1}-z_{n}||-|| x_{n+1}-x_{n}||\right)$ $\leq$. Then, $\lim _{n \rightarrow \infty}|| z_{n}-x_{n}||=0$. 
Lemma 2.3. $[10,11]$. Let $\left\{s_{n}\right\}$ be a sequence of non-negative real numbers satisfying

$$
s_{n+1} \leq\left(1-\lambda_{n}\right) s_{n}+\lambda_{n} \delta_{n}+\gamma_{n}, \quad n \geq 0,
$$

where $\left\{\lambda_{n}\right\}$ and $\left\{\gamma_{n}\right\}$ satisfy the following conditions: (i) $\left\{\lambda_{n}\right\} \subset[0,1]$ and $\sum_{n=0}^{\infty} \lambda_{n}=\infty$, (ii) $\lim \sup _{n \rightarrow \infty}$ or $\sum_{n=0}^{\infty} \lambda_{n} \delta_{n}<\infty$, (iii) $\gamma_{n} \geq 0(n \geq 0), \sum_{n=0}^{\infty} \gamma_{n}<\infty$. Then $\lim _{n \rightarrow \infty} S_{n}=0$.

Lemma 2.4. [12]. Let $\left\{a_{n}\right\}$ and $\left\{b_{n}\right\}$ be sequences of nonnegative real numbers such that $\sum_{n=0}^{\infty} b_{n}<\infty$ and $a_{n+1} \leq a_{n}+b_{n}$ for all $n \geq 0$. Then $\lim _{n \rightarrow \infty} a_{n}$ exists.

Lemma 2.5. [13]. Let $F$ be a k-Lipschitzian and $\eta$-strongly monotone operator on a Hilbert space $H$ with $0<\eta \leq k$ and $0<t<\eta / k^{2}$. Then $S=(I-t F): H \rightarrow H$ is a contraction with contraction coefficient $\tau_{t}=\sqrt{1-t\left(2 \eta-t k^{2}\right)}$.

Let $\left\{T_{i}: H \rightarrow H\right\}$. be a family of infinitely nonexpansive mappings, $\left\{\xi_{i}\right\}$ be a real sequence such that $0<\xi_{i} \leq b<1, \forall i \geq 1$. For any $n \geq 1$, define a mapping $W_{n}: H \rightarrow H$ as follows:

$$
\left\{\begin{array}{l}
U_{n, n+1}=I \\
U_{n, n}=\xi_{n} T_{n} U_{n, n+1}+\left(1-\xi_{n}\right) I \\
U_{n, n-1}=\xi_{n-1} T_{n-1} U_{n, n}+\left(1-\xi_{n-1}\right) I \\
\cdots \\
U_{n, k}=\xi_{k} T_{k} U_{n, k+1}+\left(1-\xi_{k}\right) I \\
U_{n, k-1}=\xi_{k-1} T_{k-1} U_{n, k}+\left(1-\xi_{k-1}\right) I \\
\cdots \\
U_{n, 2}=\xi_{2} T_{2} U_{n, 3}+\left(1-\xi_{2}\right) I \\
W_{n}=U_{n, 1}=\xi_{1} T_{1} U_{n, 2}+\left(1-\xi_{1}\right) I
\end{array}\right.
$$

Such a mapping $W_{n}: H \rightarrow H$ is called a $W$-mapping generated by $T_{n}, T_{n-1}, \ldots, T_{1}$ and $\xi_{n}, \xi_{n-1}, \ldots, \xi_{1}$.

We have the following crucial conclusion concerning $W_{n}$. We can find them in [14-17]. Now we only need the following similar version in Hilbert spaces:

Lemma 2.6. Let $H$ be a real Hilbert space, $\left\{T_{i}: H \rightarrow H\right\}$ be a family of infinitely nonexpansive mappings with $\cap_{i=1}^{\infty} F\left(T_{i}\right) \neq \emptyset,\left\{\xi_{i}\right\}$ be a real sequence such that $0<\xi_{i} \leq b<1$, $\forall i \geq 1$. Then,

(1) $W_{n}$ is a nonexpansive and $F\left(W_{n}\right)=\cap_{i=1}^{n} F\left(T_{i}\right)$ for each $n \geq 1$;

(2) For every $x \in H$ and $k \in N$, the limit $\lim _{n \rightarrow \infty} U_{n, k} x$ exists;

(3) If we define a mapping $W: H \rightarrow H$ as $W x=\lim _{n \rightarrow 1} W_{n} x$, and $W_{n} x=\lim _{n \rightarrow \infty}$ $U_{n, 1} x$, for every $\in H$, then, $F(W)=\cap_{i=1}^{\infty} F\left(T_{i}\right)$;

(4) For any bounded sequence $\left\{x_{n}\right\} \subset H$, we have $\lim _{n \rightarrow \infty}\left\|W x_{n}-W_{n} x_{n}\right\|=0$.

\section{Main results}

Let $F$ be a $k$-Lipschitzian and $\eta$-strongly monotone operator on $H$ with $0<k, T: H \rightarrow$ $H$ be a nonexpansive mapping. Let $t \in\left(0, \eta / k^{2}\right)$ and $\tau_{t}=\sqrt{1-t\left(2 \eta-t k^{2}\right)}$, and consider a mapping $S_{\mathrm{t}}$ on $H$ defined by

$$
S_{t} x=T[(I-t F) x], \quad x \in H .
$$


It is easy to see that $S_{t}$ is a contraction. Indeed, from Lemma 2.5, we have

$$
\begin{aligned}
\left\|S_{t} x-S_{t} y\right\| & \leq\|T[(I-t F) x]-T[(I-t F) y]\| \\
& \leq\|(I-t F) x-(I-t F) y\| \\
& \leq \tau_{t}\|x-y\|
\end{aligned}
$$

for all $x, y \in H$. Hence, it has a unique fixed point, denoted as $x_{t}$, which uniquely solves the fixed point equation

$$
x_{t}=T\left[(I-t F) x_{t}\right], \quad x_{t} \in H .
$$

Theorem 3.1. Let $H$ be a real Hilbert space. Let $T: H \rightarrow H$ be a nonexpansive mapping such that $F(T) \neq \varnothing$,. Let $F$ be a $k$-Lipschitzian and $\eta$-strongly monotone operator on $H$ with $0<\eta \leq k$. For each $t \in\left(0, \eta / k^{2}\right)$, let the net $\left\{x_{t}\right\}$ be generated by (3.1). Then, as $t \rightarrow 0$, the net $\left\{x_{t}\right\}$ converges strongly to a fixed point $x^{*}$ of $T$ which solves the variational inequality:

$$
\left\langle F x^{*}, x^{*}-u\right\rangle \leq 0, \quad \forall u \in F(T) .
$$

Proof. We first show the uniqueness of a solution of the variational inequality (3.2), which is indeed a consequence of the strong monotonicity of $F$. Suppose $x^{*} \in F(T)$ and $\tilde{x} \in F(T)$ both are solutions to (3.2), then

$$
\left\langle F x^{*}, x^{*}-\tilde{x}\right\rangle \leq 0,
$$

and

$$
\left\langle F \tilde{x}, \tilde{x}-x^{*}\right\rangle \leq 0
$$

Adding up (3.3) and (3.4) yields

$$
\left\langle F x^{*}-F \tilde{x}, x^{*}-\tilde{x}\right\rangle \leq 0 .
$$

The strong monotonicity of $F$ implies that $x^{*}=\tilde{x}$ and the uniqueness is proved. Later, we use $x^{*} \in F(T)$ to denote the unique solution of (3.2).

Next, we prove that $\left\{x_{t}\right\}$ is bounded. Take $u \in F(T)$, from (3.1) and using Lemma 2.5 , we have

$$
\begin{aligned}
\left\|x_{t}-u\right\| & =\left\|T\left[(I-t F) x_{t}\right]-T u\right\| \\
& \leq\left\|(I-t F) x_{t}-u\right\| \\
& \leq\left\|(I-t F) x_{t}-(I-t F) u-t F u\right\| \\
& \leq\left\|(I-t F) x_{t}-(I-t F) u\right\|+t\|F u\| \\
& \leq \tau_{t}\left\|x_{t}-u\right\|+t\|F u\|,
\end{aligned}
$$

that is,

$$
\left\|x_{t}-u\right\| \leq \frac{t}{1-\tau_{t}}\|F u\| .
$$

Observe that

$$
\lim _{t \rightarrow 0^{+}} \frac{t}{1-\tau_{t}}=\frac{1}{\eta} .
$$


From $t \rightarrow 0$, we may assume, without loss of generality, that $t \leq \frac{\eta}{k^{2}}-\varepsilon$, where is a arbitrarily small positive number. Thus, we have $\frac{t}{1-\tau_{t}}$ is continuous, $\forall t \in\left[0, \frac{\eta}{k^{2}}-\varepsilon\right]$. Therefore, we obtain

$$
\sup \left\{\frac{t}{1-\tau_{t}}: t \in\left(0, \frac{\eta}{k^{2}}-\varepsilon\right]\right\}<+\infty \text {. }
$$

From (3.5) and (3.6), we have that $\left\{x_{t}\right\}$ is bounded and so is $\left\{F x_{t}\right\}$.

On the other hand, from (3.1), we obtain

$$
\left\|x_{t}-T x_{t}\right\|=\left\|T\left[(I-t F) x_{t}\right]-T x_{t}\right\| \leq\left\|(I-t F) x_{t}-x_{t}\right\|=t\left\|F x_{t}\right\| \rightarrow 0(t \rightarrow 0) .
$$

To prove that $x_{t} \rightarrow x^{*}$. For a given $u \in F(T)$, using Lemma 2.5, we have

$$
\begin{aligned}
\left\|x_{t}-u\right\|^{2} & =\left\|T\left[(I-t F) x_{t}\right]-T u\right\|^{2} \\
& \leq\left\|(I-t f) x_{t}-(I-t F) u-t F u\right\|^{2} \\
& \leq \tau_{t}^{2}\left\|x_{t}-u\right\|^{2}+t^{2}\|F u\|^{2}+2 t\left\langle(I-t F) u-(I-t F) x_{t}, F u\right\rangle \\
& \leq \tau_{t}\left\|x_{t}-u\right\|^{2}+t^{2}\|F u\|^{2}+2 t\left\langle u-x_{t}, F u\right\rangle+2 t^{2}\left\langle F x_{t}-F u, F u\right\rangle \\
& \leq \tau_{t}\left\|x_{t}-u\right\|^{2}+t^{2}\|F u\|^{2}+2 t\left\langle u-x_{t}, F u\right\rangle+2 t^{2} k\left\|x_{t}-u\right\|\|F u\| .
\end{aligned}
$$

Therefore,

$$
\left\|x_{t}-u\right\|^{2} \leq \frac{t^{2}}{1-\tau_{t}}\left\|F u^{2}\right\|+\frac{2 t}{1-\tau_{t}}\left\langle u-x_{t}, F u\right\rangle+\frac{2 t^{2} k}{1-\tau_{t}}\left\|x_{t}-u\right\|\|F u\| .
$$

From $\tau_{t}=\sqrt{1-t\left(2 \eta-t k^{2}\right)}$, we have $\lim _{t \rightarrow 0} \frac{t^{2}}{1-\tau_{t}}=0$ and $\lim _{t \rightarrow 0} \frac{2 t^{2} k}{1-\tau_{t}}=0$.

Observe that, if $x_{t} \rightarrow u$, we have $\lim _{t \rightarrow 0} \frac{2 t}{1-\tau_{t}}\left\langle u-x_{t}, F u\right\rangle=0$.

Since $\left\{x_{t}\right\}$ is bounded, we see that if $\left\{t_{n}\right\}$ is a sequence in $\left(0, \frac{\eta}{k^{2}}-\varepsilon\right]$ such that $t_{n} \rightarrow 0$ and $x_{t_{n}} \rightarrow \tilde{x}$, then by (3.8), we see $x_{t_{n}} \rightarrow \tilde{x}$. Moreover, by (3.7) and using Lemma 2.1, we have $\tilde{x} \in F(T)$. We next prove that $\tilde{x}$ solves the variational inequality (3.2). From (3.1) and $u \in F(T)$, we have

$$
\begin{aligned}
\left\|x_{t}-u\right\|^{2} & \leq \|(I-t F) x_{t}-u^{2} \\
& =\left\|x_{t}-u^{2}\right\|+t^{2}\left\|F x_{t}\right\|^{2}-2 t\left\langle F x_{t}, x_{t}-u\right\rangle,
\end{aligned}
$$

that is,

$$
\left\langle F x_{t}, x_{t}-u\right\rangle \leq \frac{t}{2}\left\|F x_{t}\right\|^{2}
$$

Now replacing $t$ in (3.9) with $t_{n}$ and letting $n \rightarrow_{\infty}$, we have

$$
\langle F \tilde{x}, \tilde{x}-u\rangle \leq 0 .
$$

That is $\tilde{x} \in F(T)$ is a solution of (3.2), hence $\tilde{x}=x^{*}$ by uniqueness. In a nutshell, we have shown that each cluster point of $\left\{x_{t}\right\}$ (at $t \rightarrow 0$ ) equals $x^{*}$. Therefore, $x_{t} \rightarrow x^{*}$ as $t \rightarrow 0$.

Theorem 3.2. Let $H$ be a real Hilbert space. Let $F$ be a $k$-Lipschitzian and $\eta$-strongly monotone operator on $H$ with $0<\eta \leq k$. Let $\left\{T_{n}\right\}_{n=1}^{\infty}: H \rightarrow$ Hbe an infinite family of 
nonexpansive mappings such that $\cap_{n=1}^{\infty} F\left(T_{n}\right) \neq \emptyset$ Øand $W_{n}$ be a $W$-mapping defined by (2.3). Let $\left\{\lambda_{n}\right\}$ be a sequence in $[0, \infty)$ and $\left\{\alpha_{n}\right\}$ be a sequence in $[0,1]$, $\varepsilon$ be a arbitrarily small positive number. Assume that the control conditions (C2), (C1)', and (C5)' hold for $\left\{\lambda_{n}\right\}$ and $\left\{\alpha_{n}\right\}$,

$(C 1)^{\prime}: 0<\lambda_{n} \leq \frac{\eta}{k^{2}}-\varepsilon, \forall n \geq n_{0}$ for some integer $n_{0} \geq 0$, and

(C5) ‘: $0<\gamma \leq \lim \inf _{n \rightarrow \infty} \alpha_{n} \lim \sup _{n \rightarrow \infty} \alpha_{n}<1$ for some $\gamma \in(0,1)$.

For $x_{0} \in H$ arbitrarily, let the sequence $\left\{y_{n}\right\}$ be generated by (1.2). Then,

$$
y_{n} \rightarrow x^{*} \Leftrightarrow \lambda_{n} F\left(x_{n}\right) \rightarrow 0(n \rightarrow \infty),
$$

where $x^{*} \in \cap_{n=1}^{\infty} F\left(T_{n}\right)$ solves the variational inequality

$$
\left\langle F x^{*}, x^{*}-u\right\rangle \leq 0, \quad u \in \cap_{n=1}^{\infty} F\left(T_{n}\right) .
$$

Proof. On the one hand, suppose that $\lambda_{n} F\left(x_{n}\right) \rightarrow 0(n \rightarrow \infty)$. We proceed with the following steps:

Step 1. We claim that $\left\{x_{n}\right\}$ is bounded. In fact, let $u \in \cap_{n=1}^{\infty} F\left(T_{n}\right)$, from (1.2), (C1)' and using Lemma 2.5 , we have

$$
\begin{aligned}
\left\|y_{n}-u\right\| & =\left\|x_{n}-\lambda_{n} F\left(x_{n}\right)-u\right\| \\
& \leq\left\|\left(I-\lambda_{n} F\right) x_{n}-\left(I-\lambda_{n} F\right) u-\lambda_{n} F u\right\| \\
& \leq \tau_{\lambda_{n}}\left\|x_{n}-u\right\|+\lambda_{n}\|F u\|,
\end{aligned}
$$

$\forall n \geq n_{0}$ for some integer $n_{0} \geq 0$, where $\tau_{\lambda_{n}}=\sqrt{1-\lambda_{n}\left(2 \eta-\lambda_{n} k^{2}\right)} \in(0,1)$. Then, from (1.2) and (3.10), we obtain

$$
\begin{aligned}
\left\|x_{n+1}-u\right\| & =\left\|\left(1-\alpha_{n}\right)\left(y_{n}-u\right)+\alpha_{n}\left(W_{n} y_{n}-u\right)\right\| \\
& \leq\left\|y_{n}-u\right\| \\
& \leq\left[1-\left(1-\tau_{\lambda_{n}}\right)\right]\left\|x_{n}-u\right\|+\lambda_{n}\|F u\| \\
& \leq \max \left\{\left\|x_{n}-u\right\|, \frac{\left\|\lambda_{n} F u\right\|}{1-\tau_{\lambda_{n}}}\right\} .
\end{aligned}
$$

By induction, we have

$$
\left\|x_{n}-u\right\| \leq \max \left\{\left\|x_{0}-u\right\|, M_{1}\|F u\|\right\},
$$

$\forall n \geq n_{0}$ for some integer $n_{0} \geq 0$, where $M_{1}=\sup \left\{\frac{\lambda_{n}}{1-\tau_{\lambda_{n}}}: 0<\lambda_{n} \leq \frac{\eta}{k^{2}}-\varepsilon\right\}<+\infty$.

Therefore, $\left\{x_{n}\right\}$ is bounded. We also obtain that $\left\{y_{n}\right\},\left\{W_{n} y_{n}\right\}$ and $\left\{F x_{n}\right\}$ are bounded.

Step 2. We claim that $\lim _{n \rightarrow \infty}|| x_{n+1}-x_{n} \|=0$. To this end, define $x_{n+1}=\left(1-\alpha_{n}\right) x_{n}$ $+\alpha_{n} z_{n}$. We observe that

$$
\begin{aligned}
\left\|z_{n+1}-z_{n}\right\| & =\left\|\frac{x_{n+2}-\left(1-\alpha_{n+1}\right) x_{n+1}}{\alpha_{n+1}}-\frac{x_{n+1}-\left(1-\alpha_{n}\right) x_{n}}{\alpha_{n}}\right\| \\
& \leq\left\|\frac{\left(1-\alpha_{n+1}\right) y_{n+1}+\alpha_{n+1} W_{n+1} y_{n+1}-\left(1-\alpha_{n+1}\right) x_{n+1}}{\alpha_{n+1}}-\frac{\left(1-\alpha_{n}\right) y_{n}+\alpha_{n} W_{n} y_{n}-\left(1-\alpha_{n}\right) x_{n}}{\alpha_{n}}\right\| \\
& \leq\left\|\frac{\alpha_{n+1} W_{n+1} y_{n+1}-\left(1-\alpha_{n+1}\right) \lambda_{n+1} F\left(x_{n+1}\right)}{\alpha_{n+1}}-\frac{\alpha_{n} W_{n} y_{n}-\left(1-\alpha_{n}\right) \lambda_{n} F\left(x_{n}\right)}{\alpha_{n}}\right\| \\
& \leq \frac{1-\alpha_{n+1}}{\alpha_{n+1}}\left\|\lambda_{n+1} F\left(x_{n+1}\right)\right\|+\frac{1-\alpha_{n}}{\alpha_{n}}\left\|\lambda_{n} F\left(x_{n}\right)\right\|+\left\|W_{n+1} y_{n+1}-W_{n} y_{n}\right\| \\
& \leq \frac{1-\gamma}{\gamma}\left\|\lambda_{n+1} F\left(x_{n+1}\right)\right\|+\frac{1-\gamma}{\gamma}\left\|\lambda_{n} F\left(x_{n}\right)\right\|+\left\|W_{n+1} y_{n+1}-W_{n} y_{n}\right\| .
\end{aligned}
$$


From (2.3), for $u \in \cap_{n=1}^{\infty} F\left(T_{n}\right)$, we have

$$
\begin{aligned}
\left\|W_{n+1} y_{n}-W_{n} y_{n}\right\| & =\left\|\xi_{1} T_{1} U_{n+1,2} y_{n}-\xi_{1} T_{1} U_{n, 2} y_{n} n\right\| \\
& \leq \xi_{1}\left\|U_{n+1,2} y_{n}-U_{n, 2} y_{n} n\right\| \\
& =\xi_{1}\left\|\xi_{2} T_{2} U_{n+1,3} y_{n}-\xi_{2} T_{2} U_{n, 3} y_{n}\right\| \\
& \leq \xi_{1} \xi_{2}\left\|U_{n+1,3} y_{n}-U_{n, 3} y_{n}\right\| \\
& \leq \cdots \\
& \leq \xi_{1} \xi_{2} \cdots \xi_{n}\left\|U_{n+1, n+1} y_{n}-U_{n, n+1} y_{n}\right\| \\
& =\xi_{1} \xi_{2} \cdots \xi_{n}\left\|\xi_{n+1} T_{n+1} y_{n}+\left(1-\xi_{n+1}\right) y_{n}-y_{n}\right\| \\
& \leq\left(\prod_{i=1}^{n+1} \xi_{i}\right)\left(\left\|T_{n+1} y_{n}-u\right\|+\left\|u-y_{n}\right\|\right) \\
& \leq\left(\prod_{i=1}^{n+1} \xi_{i}\right)\left(2\left\|y_{n}-u\right\|\right) \\
& \leq M_{2} \prod_{i=1}^{n+1} \xi_{i}
\end{aligned}
$$

where $M_{2}=\sup \left\{2\left\|y_{n}-u\right\|, n \geq 0\right\}$. By (1.2) and (3.12), we have

$$
\begin{aligned}
\left\|W_{n+1} y_{n+1}-W_{n} y_{n}\right\| & \leq\left\|W_{n+1} y_{n+1}-W_{n+1} y_{n}\right\|+\left\|W_{n+1} y_{n}-W_{n} y_{n}\right\| \\
& \leq\left\|y_{n+1}-y_{n}\right\|+\left\|W_{n+1} y_{n}-W_{n} y_{n}\right\| \\
& \leq\left\|x_{n+1}-\lambda_{n+1} F\left(x_{n+1}\right)-x_{n}+\lambda_{n} F\left(x_{n}\right)\right\|+M_{2} \prod_{i=1}^{n+1} \xi_{i} \\
& \leq\left\|x_{n+1}-x_{n}\right\|+\left\|\lambda_{n+1} F\left(x_{n+1}\right)\right\|+\left\|\lambda_{n} F\left(x_{n}\right)\right\|+M_{2} \prod_{i=1}^{n+1} \xi_{i} .
\end{aligned}
$$

Substituting (3.13) into (3.11), we have

$$
\begin{aligned}
\left\|z_{n+1}-z_{n}\right\| \leq & \frac{1-\gamma}{\gamma}\left\|\lambda_{n+1} F\left(x_{n+1}\right)\right\|+\frac{1-\gamma}{\gamma}\left\|\lambda_{n} F\left(x_{n}\right)\right\|+\left\|x_{n+1}-x_{n}\right\|+\left\|\lambda_{n+1} F\left(x_{n+1}\right)\right\| \\
& +\left\|\lambda_{n} F\left(x_{n}\right)\right\|+M_{2} \prod_{i=1}^{n+1} \xi_{i} \\
= & \frac{1}{\gamma}\left\|\lambda_{n+1} F\left(x_{n+1}\right)\right\|+\frac{1}{\gamma}\left\|\lambda_{n} F\left(x_{n}\right)\right\|+\left\|x_{n+1}-x_{n}\right\|+M_{2} \prod_{i=1}^{n+1} \xi_{i}
\end{aligned}
$$

that is,

$$
\left\|z_{n+1}-z_{n}\right\|-\left\|x_{n+1}-x_{n}\right\| \leq \frac{1}{\gamma}\left\|\lambda_{n+1} F\left(x_{n+1}\right)\right\|+\frac{1}{\gamma}\left\|\lambda_{n} F\left(x_{n}\right)\right\|+M_{2} \prod_{i=1}^{n+1} \xi_{i} .
$$

Observing $\lambda_{n} F\left(x_{n}\right) \rightarrow 0(n \rightarrow \infty)$ and $0<\xi_{i} \leq b<1$, it follows that

$$
\limsup _{n \rightarrow \infty}\left(\left\|z_{n+1}-z_{n}\right\|-\left\|x_{n+1}-x_{n}\right\|\right) \leq 0 \text {. }
$$

By (C5)' and using Lemma 2.2, we have $\lim _{n \rightarrow \infty}|| z_{n}-x_{n} \|=0$. Therefore,

$$
\lim _{n \rightarrow \infty}\left\|x_{n+1}-x_{n}\right\|=\lim _{n \rightarrow \infty} \alpha_{n}\left\|z_{n}-x_{n}\right\|=0 .
$$


Step 3. We claim that $\lim _{n \rightarrow \infty}\left\|x_{n}-W_{n} x_{n}\right\|=0$. Observe that

$$
\begin{aligned}
\left\|x_{n}-W_{n} x_{n}\right\| & \leq\left\|x_{n}-x_{n+1}\right\|+\left\|x_{n+1}-W_{n} x_{n}\right\| \\
& \leq\left\|x_{n}-x_{n+1}\right\|+\left(1-\alpha_{n}\right)\left\|y_{n}-W_{n} x_{n}\right\|+\alpha_{n}\left\|W_{n} y_{n}-W_{n} x_{n}\right\| \\
& \leq\left\|x_{n}-x_{n+1}\right\|+\left(1-\alpha_{n}\right)\left\|y_{n}-x_{n}\right\|+\left(1-\alpha_{n}\right)\left\|x_{n}-W_{n} x_{n}\right\|+\alpha_{n}\left\|y_{n}-x_{n}\right\| \\
& =\left\|x_{n}-x_{n+1}\right\|+\left\|y_{n}-x_{n}\right\|+\left(1-\alpha_{n}\right)\left\|x_{n}-W_{n} x_{n}\right\|,
\end{aligned}
$$

that is,

$$
\begin{aligned}
\left\|x_{n}-W_{n} x_{n}\right\| & \leq \frac{1}{\alpha_{n}}\left(\left\|x_{n+1}-x_{n}\right\|+\left\|y_{n}-x_{n}\right\|\right) \\
& \leq \frac{1}{\gamma}\left(\left\|x_{n+1}-x_{n}\right\|+\left\|\lambda_{n} F\left(x_{n}\right)\right\|\right) \rightarrow 0(n \rightarrow \infty) .
\end{aligned}
$$

Step 4. We claim that $\lim _{n \rightarrow \infty}|| x_{n}-W x_{n} \|=0$. Indeed, we have

$$
\left\|x_{n}-W x_{n}\right\| \leq\left\|x_{n}-W_{n} x_{n}\right\|+\left\|W_{n} x_{n}-W x_{n}\right\| .
$$

By (3.15), (3.16) and using Lemma 2.6, we obtain

$$
\lim _{n \rightarrow \infty}\left\|x_{n}-W x_{n}\right\|=0 \text {. }
$$

Step 5. We claim that $\lim \sup _{n \rightarrow \infty}\left\langle F x^{*}, x^{*}-x_{n}\right\rangle \leq 0$, where $x^{*}=\lim _{n \rightarrow \infty} x_{t}$ and $x_{t}$ defined by $x_{t}=W\left[(1-t F) x_{t}\right]$. Since $x_{n}$ is bounded, there exists a subsequence $\left\{x_{n_{k}}\right\}$ of $\left\{x_{n}\right\}$ which converges weakly to $\omega$. From Step 4 , we obtain $W x_{n_{k}} \rightarrow \omega$. From Lemma 2.1, we have $\omega \in F(W)$. Hence, by Theorem 3.1, we have

$$
\limsup _{n \rightarrow \infty}\left\langle F x^{*}, x^{*}-x_{n}\right\rangle=\lim _{k \rightarrow \infty}\left\langle F x^{*}, x^{*}-x_{n_{k}}\right\rangle=\left\langle F x^{*}, x^{*}-\omega\right\rangle \leq 0 .
$$

Step 6. We claim that $\left\{x_{n}\right\}$ converges strongly to $x^{*} \in \cap_{n=1}^{\infty} F\left(T_{n}\right)$. From (1.2), we have

$$
\begin{aligned}
\left\|x_{n+1}-x^{*}\right\|^{2} & \leq\left(1-\alpha_{n}\right)\left\|y_{n}-x^{*}\right\|^{2}+\alpha_{n}\left\|W_{n} y_{n}-x^{*}\right\|^{2} \\
& \leq\left\|y_{n}-x^{*}\right\|^{2} \\
& =\left\|x_{n}-\lambda_{n} F\left(x_{n}\right)-x^{*}\right\|^{2} \\
& \leq\left\|\left(I-\lambda_{n} F\right) x_{n}-\left(I-\lambda_{n} F\right) x^{*}-\lambda_{n} F x^{*}\right\|^{2} \\
& \leq \tau_{\lambda_{n}}^{2}\left\|x_{n}-x^{*}\right\|^{2}+\lambda_{n}^{2}\left\|F x^{*}\right\|^{2}+2 \lambda_{n}\left\langle\left(I-\lambda_{n} F\right) x^{*}-\left(I-\lambda_{n} F\right) x_{n}, F x^{*}\right\rangle \\
& \leq \tau_{\lambda_{n}}\left\|x_{n}-x^{*}\right\|^{2}+\lambda_{n}^{2}\left\|F x^{*}\right\|^{2}+2 \lambda_{n}\left\langle x^{*}-x_{n}, F x^{*}\right\rangle+2 \lambda_{n}\left(\lambda_{n} F x_{n}, F x^{*}\right\rangle-2 \lambda_{n}^{2}\left\|F x^{*}\right\|^{2} \\
& \leq\left[1-\left(1-\tau_{\lambda_{n}}\right)\right]\left\|x_{n}-x^{*}\right\|^{2}+2 \lambda_{n}\left\langle x^{*}-x_{n}, F x^{*}\right\rangle+2 \lambda_{n}\left\|\lambda_{n} F x_{n}\right\|\left\|F x^{*}\right\|-\lambda_{n}^{2}\left\|F x^{*}\right\|^{2} \\
& \leq\left[1-\left(1-\tau_{\lambda_{n}}\right)\right]\left\|x_{n}-x^{*}\right\|^{2}+\left(1-\tau_{\lambda_{n}}\right)\left[\frac{2 \lambda_{n}}{1-\tau_{\lambda_{n}}}\left\langle x^{*}-x_{n}, F x^{*}\right\rangle+\frac{\lambda_{n} M_{3}}{1-\tau_{\lambda_{n}}}\left\|\lambda_{n} F x_{n}\right\|\right] \\
& \leq\left[1-\left(1-\tau_{\lambda_{n}}\right)\right]\left\|x_{n}-x^{*}\right\|^{2}+\left(1-\tau_{\lambda_{n}}\right)\left[2 M_{1}\left\langle x^{*}-x_{n}, F x^{*}\right\rangle+M_{1} M_{3}\left\|\lambda_{n} F x_{n}\right\|\right],
\end{aligned}
$$

$\forall n \geq n_{0}$ for some integer $n_{0} \geq 0$, where $M_{3}=2\left\|F x^{*}\right\|$. For every $n \geq n_{0}$, put $\mu_{n}=1-\tau_{\lambda_{n}}$ and $\delta_{n}=2 M_{1}\left\langle x^{*}-x_{n}, F x^{*}\right\rangle+M_{1} M_{3}\left\|\lambda_{n} F x_{n}\right\|$. It follows that

$$
\left\|x_{n+1}-x^{*}\right\|^{2} \leq\left(1-\mu_{n}\right)\left\|x_{n}-x^{*}\right\|^{2}+\mu_{n} \delta_{n}, \quad \forall n \geq n_{0} .
$$

It is easy to see that $\sum_{n=1}^{\infty} \mu_{n}=\infty$ and $\lim \sup _{n \rightarrow \infty} \delta_{n} \leq 0$. Hence, by Lemma 2.3, the sequence $\left\{x_{n}\right\}$ converges strongly to $x^{*} \in \cap_{n=1}^{\infty} F\left(T_{n}\right)$.

Observe that

$$
\left\|y_{n}-x^{*}\right\| \leq\left\|y_{n}-x_{n}\right\|+\left\|x_{n}-x^{*}\right\| \leq\left\|\lambda_{n} F\left(x_{n}\right)+\right\| x_{n}-x^{*} \| \rightarrow 0(n \rightarrow \infty) .
$$

It follows that the sequence $\left\{y_{n}\right\}$ converges strongly to $x^{*} \in \cap_{n=1}^{\infty} F\left(T_{n}\right)$. From $x^{*}=$ $\lim _{t \rightarrow 0} x_{t}$ and Theorem 3.1, we have $x^{*}$ is the unique solution of the variational inequality: $\left\langle F x^{*}, x^{*}-u\right\rangle \leq 0, \forall u \in \cap_{n=1}^{\infty} F\left(T_{n}\right)$. 
On the other hand, suppose that $y_{n} \rightarrow x^{*} \in \cap_{n=1}^{\infty} F\left(T_{n}\right)$ as $n \rightarrow \infty$, where $x^{*} \in \cap_{n=1}^{\infty} F\left(T_{n}\right)$ solves the variational inequality:

$$
\left\langle F x^{*}, x^{*}-u\right\rangle \leq 0, \quad u \in \cap_{n=1}^{\infty} F\left(T_{n}\right) .
$$

From (1.2), we have

$$
\begin{aligned}
\left\|x_{n+1}-x^{*}\right\| & =\left\|\left(1-\alpha_{n}\right)\left(y_{n}-x^{*}\right)+\alpha_{n}\left(W_{n} y_{n}-x^{*}\right)\right\| \\
& \leq\left(1-\alpha_{n}\right)\left\|y_{n}-x^{*}\right\|+\alpha_{n}\left\|y_{n}-x^{*}\right\| \\
& =\| y_{n}-x^{*} \rightarrow 0(n \rightarrow \infty),
\end{aligned}
$$

that is, $x_{n} \rightarrow x^{*} \in \cap_{n=1}^{\infty} F\left(T_{n}\right)$. Again from (1.2), we obtain that

$$
\left\|\lambda_{n} F\left(x_{n}\right)\right\|=\left\|y_{n}-x_{n}\right\| \leq\left\|y_{n}-x^{*}\right\|+\left\|x_{n}-x^{*}\right\|
$$

Since $y_{n} \rightarrow x^{*} \in \cap_{n=1}^{\infty} F\left(T_{n}\right)$ and $x_{n} \rightarrow x^{*} \in \cap_{n=1}^{\infty} F\left(T_{n}\right)$, we get $\lambda_{n} F\left(x_{n}\right) \rightarrow 0$. This completes the proof.

Remark 3.3. It is clear that condition (C1)' is strictly weaker than condition (C1). In the meantime, condition (C5)' is also strictly weaker than condition (C5).

Corollary 3.4. (Yao et al. [5, Theorem 3.2]). Let $H$ be a real Hilbert space. Let F : H $\rightarrow H$ be $k$-Lipschitzian and $\eta$-strongly monotone operator with $k \in[1, \infty)$ and $\eta \in(0$, 1). Let $\left\{T_{n}\right\}_{n=1}^{\infty}: H \rightarrow$ Hbe an infinite family of nonexpansive mappings such that $\cap_{n=1}^{\infty} F\left(T_{n}\right) \neq \emptyset$ and $\left\{W_{n}\right\}$ be $W$-mapping defined by (2.3). Let $\left\{\lambda_{n}\right\}$ be a sequence in $[0$, $\infty)$ and $\left\{\alpha_{n}\right\}$ be a sequence in $[0,1]$. Assume that

(C1) $\lim _{n \rightarrow 1} \lambda_{n}=0$;

(C2) $\sum_{n=0}^{\infty} \lambda_{n}=\infty$;

(C5) $\alpha_{n} \in\left[\gamma, \frac{1}{2}\right]$ for some $\gamma>0$.

Then, the sequence $\left\{x_{n}\right\}$ and $\left\{y_{n}\right\}$ generated by (1.2) converge strongly to $x^{*} \in \cap_{n=1}^{\infty} F\left(T_{n}\right)$, which solves the following variational inequality $\left\langle F x^{*}, x^{*}-x\right\rangle \leq 0$, $x^{*} \in \cap_{n=1}^{\infty} F\left(T_{n}\right)$.

Proof. Since $\lim _{n \rightarrow \infty} \lambda_{n}=0$, it is easy to see that $\lambda_{n} \leq \frac{\eta}{k^{2}}-\varepsilon, \forall n \geq n_{0}$ for some integer $n_{0} \geq 0$. Without loss of generality, we assume that $0<\lambda_{n} \leq \frac{\eta}{k^{2}}-\varepsilon, \forall n \geq n_{0}$ for some integer $n_{0} \geq 0$. Repeating the same argument as in the proof of Theorem 3.2, we know that $\left\{x_{n}\right\}$ is bounded, and so are the sequence $\left\{y_{n}\right\}$ and $\left\{F\left(x_{n}\right)\right\}$. Therefore, we have $\lambda_{n} F\left(x_{n}\right) \rightarrow 0$.

From $\alpha_{n} \in\left[\gamma, \frac{1}{2}\right]$ for some $\gamma>0$, we have $0<\gamma \leq \lim \inf _{n \rightarrow \infty} \alpha_{n} \leq \lim \sup _{n \rightarrow \infty} \alpha_{n}<$ 1 for some $\gamma \in(0,1)$. Therefore, all conditions of Theorem 3.2 are satisfied. Hence, using Theorem 3.2, we have that $\left\{y_{n}\right\}$ converges strongly to $x^{*} \in \cap_{n=1}^{\infty} F\left(T_{n}\right)$ which solves the following variational inequality $\left\langle F x^{*}, x^{*}-x\right\rangle \leq 0, x^{*} \in \cap_{n=1}^{\infty} F\left(T_{n}\right)$. It follows from (3.17) that $\left\{x_{n}\right\}$ also converges strongly to $x^{*} \in \cap_{n=1}^{\infty} F\left(T_{n}\right)$. This completes the proof. 
Remark 3.5. Theorem 3.2 is more general than Theorem 3.2 of Yao et al. [5]. The following example shows that all conditions of Theorem 3.2 are satisfied. However, the conditions $\lambda_{n} \rightarrow 0, \eta \in(0,1)$ and $\alpha_{n} \in\left[\gamma, \frac{1}{2}\right]$ for some $\gamma>0$ in [5, Theorem 3.2] are not satisfied.

Example 3.6. Let $H=R$ the set of real numbers and $T_{n} \equiv T$. Define a nonexpansive mapping $T: H \rightarrow H$ and an operator $F: H \rightarrow H$ as follows:

$$
T x=0 \quad \text { and } \quad F(x)=x, \quad \forall x \in R .
$$

It is easy to see that $F(T)=\{0\}, \cap_{n=1}^{\infty} F\left(T_{n}\right)=\{0\}$ and $W_{n} x=\left(1-\xi_{1}\right) x, \forall x \in R$. Let $\xi_{1}=\frac{1}{2}$, we have $W_{n} x=\frac{1}{2} x, \forall x \in R$. Given sequences $\left\{\alpha_{n}\right\}$ and $\left\{\lambda_{n}\right\}: \alpha_{n}=\frac{2}{3}, \lambda_{n}=\frac{1}{2}$ for all $n \geq 0$. For an arbitrary $x_{0} \in H$, let $\left\{x_{n}\right\}$ defined as follows:

$$
\left\{\begin{array}{l}
y_{n}=x_{n}-\lambda_{n} F\left(x_{n}\right), \\
x_{n+1}=\left(1-\alpha_{n}\right) y_{n}+\alpha_{n} W_{n} y_{n}, n \geq 0,
\end{array}\right.
$$

that is,

$$
\begin{aligned}
& y_{n}=x_{n}-\lambda_{n} F\left(x_{n}\right)=\frac{1}{2} x_{n} \\
& x_{n+1}=\frac{1}{3} y_{n}+\frac{2}{3} W_{n} y_{n}=\frac{2}{3} y_{n}=\frac{1}{3} x_{n}, \quad n \geq 0 .
\end{aligned}
$$

Observe that for all $n \geq 0$,

$$
\left\|x_{n+1}-0\right\|=\frac{1}{3}\left\|x_{n}-0\right\| .
$$

Hence, we have $\left\|x_{n+1}-0\right\|=\left(\frac{1}{3}\right)^{n+1}\left\|x_{0}-0\right\|$ for all $n \geq 0$. This implies that $\left\{x_{n}\right\}$ converges strongly to $0 \in \cap_{n=1}^{\infty} F\left(T_{n}\right)$. Since $\left\|y_{n}-0\right\|=\frac{1}{2}\left\|x_{n}\right\| \rightarrow 0$, we have that $\left\{y_{n}\right\}$ converges strongly to $0 \in \cap_{n=1}^{\infty} F\left(T_{n}\right)$.

Observe that $\langle F(0), 0-u\rangle \leq 0, u \in \cap_{n=1}^{\infty} F\left(T_{n}\right)$, that is, 0 is the solution of the variational inequality $\left\langle F x^{*}, x^{*}-u\right\rangle \leq 0, u \in \cap_{n=1}^{\infty} F\left(T_{n}\right)$.

Finally, we have

$$
\left\|\lambda_{n} F\left(x_{n}\right)\right\|=\frac{1}{2}\left\|x_{n}\right\| \rightarrow 0(n \rightarrow \infty) .
$$

By $F(x)=x$, we have $\eta=k=1$. Furthermore, it is easy to see that the following hold true:

(B1) $0<\lambda_{n}=\frac{1}{2} \leq 1-\varepsilon, \forall n \geq n_{0}$ for some integer $n_{0} \geq 0$;

(B2) $\sum_{n=0}^{\infty} \lambda_{n}=\sum_{n=0}^{\infty} \frac{1}{2}=\infty$;

(B3) $0<\frac{1}{2} \leq \liminf _{n \rightarrow \infty} \alpha_{n}=\frac{2}{3}=\lim \sup _{n \rightarrow \infty} \alpha_{n}<1$ for some constant $\gamma=\frac{1}{2}$. 
Hence, there is no doubt that all conditions of Theorem 3.2 are satisfied. Since $\lambda_{n}=\frac{1}{2}$, $\eta=1$ and $\alpha_{n} \in\left[\gamma, \frac{1}{2}\right]$, the conditions that $\lambda_{n} \rightarrow 0, \alpha_{n} \in\left[\gamma, \frac{1}{2}\right]$ for some $\gamma>0$ and $\eta \in$ $(0,1)$ of Yao et al. [5, Theorem 3.2] are not satisfied.

Next, we give a weak convergence theorem for hybrid iterative algorithm (1.2) involving an infinite family of nonexpansive mappings in a Hilbert space.

Theorem 3.7. Let $H$ be a real Hilbert space. Let $F: H \rightarrow H$ be k-Lipschitzian and $\eta$-strongly monotone operator with $0<\eta \leq k$. Let $\left\{T_{n}\right\}_{n=1}^{\infty}: H \rightarrow$ Hbe an infinite family of nonexpansive mappings such that $\cap_{n=1}^{\infty} F\left(T_{n}\right) \neq \emptyset$, and $\left\{W_{n}\right\}$ be $W$ mapping defined by (2.3). Let $\left\{\lambda_{n}\right\}$ and $\left\{\alpha_{n}\right\}$ be two sequences in $(0,1)$. Assume that

(A1) $\sum_{n=0}^{\infty} \lambda_{n}=\infty$;

(A2) $0<\lim \inf _{n \rightarrow \infty} \alpha_{n} \leq \lim \sup _{n \rightarrow \infty} \alpha_{n}<1$.

Then, the sequence $\left\{x_{n}\right\}$ and $\left\{y_{n}\right\}$ generated by (1.2) converge weakly to $x^{*} \in \cap_{n=1}^{\infty} F\left(T_{n}\right)$. Proof. From (A1), we have $0<\lambda_{n} \leq \frac{\eta}{k^{2}}-\varepsilon, \forall n \geq n_{0}$ for some integer $n_{0} \geq 0$. Repeating the same argument as in the proof of Theorem 3.2, we know that $\left\{x_{n}\right\}$ is bounded, and so are the sequences $\left\{y_{n}\right\}$ and $\left\{F\left(x_{n}\right)\right\}$. Assuming $p \in \cap_{n=1}^{\infty} F\left(T_{n}\right)$, we have

$$
\begin{aligned}
\left\|x_{n+1}-p\right\|^{2} & =\left\|\left(1-\alpha_{n}\right)\left(y_{n}-p\right)+\alpha_{n}\left(W_{n} y_{n}-p\right)\right\|^{2} \\
& =\left(1-\alpha_{n}\right)\left\|y_{n}-p\right\|^{2}+\alpha_{n}\left\|W_{n} y_{n}-p\right\|^{2}-\left(1-\alpha_{n}\right) \alpha_{n}\left\|y_{n}-W_{n} y_{n}\right\|^{2} \\
& \leq\left\|y_{n}-p\right\|^{2}-\left(1-\alpha_{n}\right) \alpha_{n}\left\|y_{n}-W_{n} y_{n}\right\|^{2} \\
& =\left\|x_{n}-p-\lambda_{n} F\left(x_{n}\right)\right\|^{2}-\left(1-\alpha_{n}\right) \alpha_{n}\left\|y_{n}-W_{n} y_{n}\right\|^{2} \\
& \leq\left[\left\|x_{n}-p\right\|+\lambda_{n}\left\|F\left(x_{n}\right)\right\|\right]^{2}-\left(1-\alpha_{n}\right) \alpha_{n}\left\|y_{n}-W_{n} y_{n}\right\|^{2} \\
& =\left\|x_{n}-p\right\|^{2}+\lambda_{n}^{2}\left\|F\left(x_{n}\right)\right\|^{2}+2 \lambda_{n}\left\|x_{n}-p\right\|\left\|F\left(x_{n}\right)\right\|-\left(1-\alpha_{n}\right) \alpha_{n}\left\|y_{n}-W_{n} y_{n}\right\|^{2} \\
& \leq\left\|x_{n}-p\right\|^{2}+M_{4}\left(\lambda_{n}^{2}+\lambda_{n}\right),
\end{aligned}
$$

where $M_{4}=\sup \left\{\left\|F\left(x_{n}\right)\right\|^{2}, 2\left\|x_{n}-p\right\|\left\|F\left(x_{n}\right)\right\|, n \geq 0\right\}$. Since $\sum_{n=0}^{\infty} \lambda_{n}=\infty$, we have $\sum_{n=0}^{\infty} \lambda_{n}^{2}<\infty$. Therefore, $\sum_{n=0}^{\infty} M_{4}\left(\lambda_{n}^{2}+\lambda_{n}\right)<\infty$. Utilizing Lemma 2.4, we deduce that $\lim _{n \rightarrow \infty}\left\|x_{n}-p\right\|$ exists. Further-more, from(3.17), we have

$$
\left(1-\alpha_{n}\right) \alpha_{n}\left\|y_{n}-W_{n} y_{n}\right\|^{2} \leq\left\|x_{n}-p\right\|^{2}-\left\|x_{n+1}-p\right\|^{2}+M_{4}\left(\lambda_{n}^{2}+\lambda_{n}\right) .
$$

Since $\lambda_{n} \rightarrow 0, \lambda_{n}^{2} \rightarrow 0$ and (A2), it follows from (3.18) that

$$
\left\|y_{n}-W_{n} y_{n}\right\| \rightarrow 0(n \rightarrow \infty) .
$$

Utilizing Lemma 2.6, we have

$$
\left\|y_{n}-W y_{n}\right\| \leq\left\|y_{n}-W_{n} y_{n}\right\|+\left\|W_{n} y_{n}-W y_{n}\right\| \rightarrow 0(n \rightarrow \infty) .
$$

Now, we show that $\omega_{w}\left(y_{n}\right) \subset F(T)$. Indeed, let $x^{*} \in \omega_{w}\left(y_{n}\right)$. Then, there exists a subsequence $\left\{y_{n_{i}}\right\}$ of $\left\{y_{n}\right\}$ such that $y_{n_{i}} \rightarrow x^{*}$. Since $\left\|y_{n}-W y_{n}\right\| \rightarrow 0$, by Lemma 2.1, we have $x^{*} \in F(W)=\cap_{n=1}^{\infty} F\left(T_{n}\right)$. 
Next, we show that $\omega_{w}\left(y_{n}\right)$ is a singleton. Indeed, let $\left\{y_{m_{j}}\right\}$ be another subsequence of $\left\{y_{n}\right\}$ such that $y_{m_{j}} \rightarrow \tilde{x}$. Then, $\tilde{x} \in \cap_{n=1}^{\infty} F\left(T_{n}\right)$. If $x^{*} \neq \tilde{x}$, then, by Opial's property of $H$, we have

$$
\begin{aligned}
\lim _{n \rightarrow \infty}\left\|y_{n}-x^{*}\right\| & =\lim _{i \rightarrow \infty}\left\|y_{n_{i}}-x^{*}\right\| \\
& <\lim _{i \rightarrow \infty}\left\|y_{n_{i}}-\tilde{x}\right\| \\
& =\lim _{j \rightarrow \infty}\left\|y_{m_{j}}-\tilde{x}\right\| \\
& <\lim _{j \rightarrow \infty}\left\|y_{m_{j}}-x^{*}\right\| \\
& =\lim _{n \rightarrow \infty}\left\|y_{n}-x^{*}\right\| .
\end{aligned}
$$

This is a contradiction. Therefore, $\omega_{w}\left(y_{n}\right)$ is a singleton. Consequently, $\left\{y_{n}\right\}$ converges weakly to $x^{*} \in \cap_{n=1}^{\infty} F\left(T_{n}\right)$. From (1.2), we have that $\left\{x_{n}\right\}$ converges weakly to $x^{*} \in \cap_{n=1}^{\infty} F\left(T_{n}\right)$. This completes the proof.

Remark 3.8. It is worth pointing out that the conditions (C1) and (C2) in [5, Theorem 3.2] are replaced by the one (A1) in Theorem 3.7. It is also worth pointing out that condition (A2) is strictly weaker than the condition (C5). The advantages of there results in this study are that weaker and fewer restrictions are imposed on parameters $\alpha_{n}, \lambda_{n}$ and $\eta$.

\section{Acknowledgements}

This study was supported by the Natural Science Foundation of Yancheng Teachers University under Grant (10YCKL022)

\section{Competing interests}

The authors declare that they have no competing interests.

Received: 24 January 2011 Accepted: 20 June 2011 Published: 20 June 2011

\section{References}

1. Stampacchia, G: Formes bilineaires coercitives sur les ensembles convexes. C.R Acad Sci Paris. 258, 4413-4416 (1964)

2. Yamada, I: The hybrid steepest descent method for the variational inequality problem over the intersection of fixed point sets of nonexpansive mappings. In: Butnariu D, Censor Y, Reich S (eds.) Stud Comput Math, vol. 8, pp. 473-504. Inherently Parallel Algorithms in Feasibility and Optimization and Their Applications (Haifa, 2000), North-Holland, Amsterdam (2001)

3. Lions, PL: Approximation de points fixes de contractions. C.R Acad Sci Paris. 284, 1357-1359 (1977)

4. $\mathrm{Xu}, \mathrm{HK}, \mathrm{Kim}, \mathrm{TH}$ : Convergence of hybrid steepest-descent methods for variational inequalities. J Optim Theory Appl. 119, 185-201 (2003)

5. Yao, YH, Noor, MN, Liou, YC: A new hybrid iterative algorithm for variational inequalities. Appl Math Comput. 216, 822-829 (2010). doi:10.1016/j.amc.2010.01.087

6. Opial, Z: Weak convergence of successive approximations for nonexpansive mappings. Bull Am Math Soc. 73, 591-597 (1967). doi:10.1090/S0002-9904-1967-11761-0

7. Takahashi, W: Introduction to Nonlinear and Convex Analysis. Yokohama Publisher, Yokohama (2009)

8. Geobel, K, Kirk, WA: Topics in Metric Fixed point Theory. In Cambridge Stud Adv Math, vol. 28, pp. 473-504.Cambridge University Press, Cambridge (1990)

9. Suzuki, T: Strong convergence of Krasnoselskii and Mann's type sequences for one parameter nonexpansive semigroups without Bochner integral. J Math Anal Appl. 35, 227-239 (2005)

10. Liu, LS: Iterative processes with errors for nonlinear strongly accretive mappings in Banach spaces. J Math Anal Appl. 194, 114-125 (1995). doi:10.1006/jmaa.1995.1289

11. Xu, H-K: Iterative algorithms for nonlinear operators. J Lond Math Soc. 66(1), 240-256 (2002). doi:10.1112/ S0024610702003332

12. Tan, KK, Xu, HK: Approximating fixed points of nonexpansive mappings by the Ishikawa iterative process. J Math Anal Appl. 178, 301-308 (1993). doi:10.1006/jmaa.1993.1309

13. Wang, S, Hu, CS: Two New Iterative Methods for a Countable Family of Nonexpansive Mappings in Hilbert Spaces. Fixed Point Theory Appl 2010, 12 (2010). (Article ID 852030)

14. Takahashi, W, Shimoji, K: Convergence theorems for nonexpansive mappings and feasibility problems. Math Comput Model. 32, 1463-1471 (2000). doi:10.1016/S0895-7177(00)00218-1

15. Shimoji, K, Takahashi, W: Strong convergence to common fixed points of infinite nonexpansive mappings and applications. Taiwan J Math. 5, 387-404 (2001) 
16. Chang, S-S: A new method for solving equilibrium problem and variational inequality problem with application to optimization. Nonlinear Anal. 70, 3307-3319 (2009). doi:10.1016/j.na.2008.04.035

17. Yao, YH, Liou, YC, Yao, JC: Convergence theorem for equilibrium problems and fixed point problems of infinite family of nonexpansive mappings. Fixed Point Theory Appl 2007, 12 (2007). (Article ID 64363)

doi:10.1186/1687-1812-2011-3

Cite this article as: Wang: Convergence and weaker control conditions for hybrid iterative algorithms. Fixed Point Theory and Applications 2011 2011:3.

Submit your manuscript to a SpringerOpen ${ }^{\odot}$ journal and benefit from:

- Convenient online submission

- Rigorous peer review

- Immediate publication on acceptance

- Open access: articles freely available online

- High visibility within the field

- Retaining the copyright to your article

Submit your next manuscript at $\boldsymbol{\nabla}$ springeropen.com 